\title{
An Evaluation of Hearing Aid Beamforming Microphone Arrays in a Noisy Laboratory Setting
}

DOI: $10.3766 /$ jaaa. 17090

Erin M. Picou*

Todd A. Ricketts*

\begin{abstract}
Background: People with hearing loss experience difficulty understanding speech in noisy environments. Beamforming microphone arrays in hearing aids can improve the signal-to-noise ratio (SNR) and thus also speech recognition and subjective ratings. Unilateral beamformer arrays, also known as directional microphones, accomplish this improvement using two microphones in one hearing aid. Bilateral beamformer arrays, which combine information across four microphones in a bilateral fitting, further improve the SNR. Early bilateral beamformers were static with fixed attenuation patterns. Recently adaptive, bilateral beamformers have been introduced in commercial hearing aids.
\end{abstract}

Purpose: The purpose of this article was to evaluate the potential benefits of adaptive unilateral and bilateral beamformers for improving sentence recognition and subjective ratings in a laboratory setting. A secondary purpose was to identify potential participant factors that explain some of the variability in beamformer benefit.

Research Design: Participants were fitted with study hearing aids equipped with commercially available adaptive unilateral and bilateral beamformers. Participants completed sentence recognition testing in background noise using three hearing aid settings (omnidirectional, unilateral beamformer, bilateral beamformer) and two noise source configurations (surround, side). After each condition, participants made subjective ratings of their perceived work, desire to control the situation, willingness to give up, and tiredness.

Study Sample: Eighteen adults (50-80 yr, $M=66.2, \sigma=8.6)$ with symmetrical mild sloping to severe hearing loss participated.

Data Collection and Analysis: Sentence recognition scores and subjective ratings were analyzed separately using generalized linear models with two within-subject factors (hearing aid microphone and noise configuration). Two benefit scores were calculated: (1) unilateral beamformer benefit (relative to performance with omnidirectional) and (2) additional bilateral beamformer benefit (relative to performance with unilateral beamformer). Hierarchical multiple linear regression was used to determine if beamformer benefit was associated with participant factors (age, degree of hearing loss, unaided speech in noise ability, spatial release from masking, and performance in omnidirectional).

Results: Sentence recognition and subjective ratings of work, control, and tiredness were better with both types of beamformers relative to the omnidirectional conditions. In addition, the bilateral beamformer offered small additional improvements relative to the unilateral beamformer in terms of sentence recognition and subjective ratings of tiredness. Speech recognition performance and subjective ratings were generally independent of noise configuration. Performance in the omnidirectional setting and pure-tone average were independently related to unilateral beamformer benefits. Those with the lowest performance or the largest degree of hearing loss benefited the most. No factors were significantly related to additional bilateral beamformer benefit.

Conclusions: Adaptive bilateral beamformers offer additional advantages over adaptive unilateral beamformers in hearing aids. The small additional advantages with the adaptive beamformer are

*Department of Hearing and Speech Sciences, Vanderbilt University Medical Center, Nashville, TN

Corresponding author: Erin M. Picou, Department of Hearing and Speech Sciences, Vanderbilt University Medical Center, Nashville, TN 37232; Email: erin.picou@vanderbilt.edu

This project was funded by Sonova and by the Dan and Margaret Maddox Charitable Fund.

Portions of this project were presented at AudiologyNow in Indianapolis, IN, April 5-8, 2017. 
comparable to those reported in the literature with static beamformers. Although the additional benefits are small, they positively affected subjective ratings of tiredness. These data suggest that adaptive bilateral beamformers have the potential to improve listening in difficult situations for hearing aid users. In addition, patients who struggle the most without beamforming microphones may also benefit the most from the technology.

Key Words: hearing aid, hearing loss, noise, self-report, speech perception

Abbreviations: $\mathrm{Cl}=$ confidence interval; $\mathrm{CST}=$ Connected Speech Test; FAPTA = frequency pure-tone average; HINT = Hearing in Noise Test; PTA = pure-tone average; QuickSIN = Quick Speech in Noise Test; RAU = rationalized arcsine units; SNR = signal-to-noise ratio; SRM = spatial release from masking

\section{INTRODUCTION}

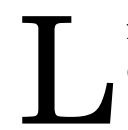
isteners with sensorineural hearing loss have difficulty understanding speech in noise (Plomp, 1986; Sherbecoe and Studebaker, 2003). Although hearing aids can improve speech understanding (Humes and Wilson, 2003; Picou et al, 2013), understanding speech in noise continues to be a primary clinical complaint. Indeed, such difficulties contribute to hearing aid satisfaction (Kochkin, 2010) and often lead to patients purchasing, but not using, hearing aids (Kochkin, 2000; McCormack and Fortnum, 2013). Consequently, hearing aid microphone technologies continue to be developed and improved to address speech understanding difficulties experienced by people with hearing loss.

One such group of microphone technologies are beamforming microphone arrays referred to herein as "beamformers." These arrays use two or more microphones to enhance directivity. The result is an improved the signalto-noise ratio (SNR) in many environments because beamformers are more sensitive to sounds originating from one direction than from a combination of all other directions (Bentler et al, 2004). Beamformers are presently applied in commercial hearing aids in unilateral or bilateral configurations. Unilateral beamformers, also called directional microphones in the hearing aid literature, combine the output of two microphones in one hearing aid. The use of unilateral beamforming in hearing aids has been studied extensively in laboratory settings. Results indicate significant improvements in speech understanding when the speech signal arrives from the front and competing noise arrives from other directions or surrounds the listener (Preves et al, 1999; ComptonConley et al, 2004; Bentler et al, 2006; Aspell et al, 2014). Although unilateral beamformers can offer large speech recognition benefits, these benefits are reduced by high reverberation (Ricketts and Dhar, 1999; Ricketts and Hornsby, 2003), large talker to listener distances that exceed the critical distance (Ricketts and Hornsby, 2003), and ceiling effects (Wu and Bentler, 2010). In addition, measured benefits are often not reflected by participant subjective preference (Walden et al, 2003; Gnewikow et al, 2009; Wu, 2010).

Bilateral beamforming is similar to unilateral beamforming, except the bilateral application uses the out- put from all four microphones present in a bilateral hearing aid fitting to create a single higher order microphone array. The output of the array is delivered to both hearing aids simultaneously. Because of the potential for significant negative spatial hearing consequences of presenting the same signal to both ears, commercially implemented bilateral beamformers balance directivity with bilateral hearing, typically by reintroducing binaural cues or by employing bilateral beamforming only in certain frequencies (Lotter and Vary, 2006). Results of laboratory investigations with commercially available bilateral beamformers indicate additional improvements in speech understanding relative to unilateral beamformers (Kompis and Dillier, 1994; Cornelis et al, 2012; Picou et al, 2014; Best et al, 2015). In addition, results reveal preserved localization ability for sounds originating within approximately $30^{\circ}-60^{\circ}$ of midline (Picou et al, 2014; Best et al, 2015). Similar to unilateral beamformers, documented speech recognition benefits do not necessarily directly result in improved subjective ratings or patient preference for bilateral beamformers (Picou et al, 2014; Best et al, 2015).

Although commercially implemented hearing aid bilateral beamformers have been shown to improve speech understanding in some environments, the additional benefits relative to unilateral beamformers have been modest (Cornelis et al, 2012; Picou et al, 2014; Best et al, 2015). For example, Picou et al (2014) evaluated sentence recognition in noise in several SNRs in two environments, an audiometric sound booth and a moderately reverberant environment. Participants were fitted with bilateral hearing aids and three levels of beamforming (mild unilateral, moderate unilateral, and bilateral). Results revealed small, nonsignificant additional benefits of the bilateral compared with the unilateral beamformer in the sound booth (approximately 4 percentage points). In the moderately reverberant environment, results revealed an additional 6-12 percentage point improvement with the bilateral relative to the moderate unilateral beamformer. Best et al (2015) reported similarly modest benefits with two different hearing aid bilateral beamformers (6-11 percentage points).

These results demonstrate that although bilateral beamformers have the potential to improve speech recognition relative to a unilateral beamformer, the 
benefits might be limited to specific environments and are likely to be modest. Like unilateral beamformers, bilateral beamformer benefit has been shown to be limited by reverberation (Welker et al, 1997), room size (Kompis and Dillier, 2001), target location (Best et al, 2015), and SNR (Picou et al, 2014), with larger benefits documented at steeper portions of the performanceintensity function and in larger rooms. An additional limitation of the first generation of commercially available bilateral beamformers is they implemented static attenuation patterns. Therefore, these early beamformers were not expected to deliver benefits for noise configurations for which they were not optimized (e.g., noise sources only arriving from $90^{\circ}$ to $270^{\circ}$ ). Consequently, better performance might be expected for adaptive unilateral beamformers than static bilateral beamformers if noise was delivered only to the sides.

One potential method of improving the benefits of bilateral beamforming may therefore be to implement the technology adaptively with the goal of actively adjusting the angles of maximum attenuation (nulls) to optimize SNR across a variety of noise configurations. Previous work with adaptive unilateral beamforming in hearing aids confirms that adaptive technology can improve speech understanding relative to a static unilateral beamformer (Kuk et al, 2005), especially when noise signals originate from the side (Ricketts and Henry, 2002; Blamey et al, 2006). The benefits of adaptive bilateral beamformers have not been previously investigated despite the potential for some similar adaptive advantages. However, it is also possible that the distorted binaural information provided by adaptive bilateral beamformers will limit potential benefits compared with their unilateral counterparts.

Although the aforementioned results generally suggest speech recognition benefits for unilateral and bilateral beamformers, the data also suggest significant variability in reported benefits. For example, using a fixed SNR sentence recognition test, $\mathrm{Wu}$ and Bentler (2010) reported laboratory unilateral beamformer benefits in the presence of noise from approximately -15 to +70 rationalized arcsine units (RAU) using auditoryonly stimuli. Others have also reported similarly large ranges in unilateral beamformer benefits (Valente et al, 1995; Ricketts and Mueller, 2000; Dhar et al, 2004; Wu, 2010; Keidser et al, 2013). As a result of this significant interparticipant variability, it has been of clinical and scientific interest to identify patient factors that might influence beamformer benefits.

\section{Predictive Factors}

\section{Unilateral Beamformers}

One might expect that age and degree of hearing loss would both be inversely related to unilateral beam- former benefit, primarily because of the influence these factors have on a listener's performance-intensity function. Age and degree of hearing loss may both limit a listener's expected ceiling performance level (Plomp, 1986; Sherbecoe and Studebaker, 2002) and thus limit the potential benefit. However, previous results generally indicate nonsignificant relationships measured in the laboratory between benefit and age (Wu, 2010). In addition, within a population of listeners with moderate hearing loss, investigators have reported nonsignificant relationships between beamformer benefit and degree of hearing loss (Jespersen and Olsen, 2003; Keidser et al, 2013), although listeners with moderate losses are generally likely to experience larger beamformer benefits than their peers with more severe hearing loss (Ricketts et al, 2005; Ricketts and Hornsby, 2006; Picou and Ricketts, 2017).

Another potential predictive factor is an individual's speech in noise performance. It has been argued that people who need a better SNR to understand speech in noise might also be more likely to benefit from a technology with potential to substantially improve the SNR (Killion and Christensen, 1998; Taylor, 2003). Consistent with this argument, Ricketts and Mueller (2000) found a significant relationship between SNR needed for $50 \%$ speech recognition in an aided omnidirectional condition and directional benefit. It is of interest clinically to evaluate whether this relationship holds for unaided speech recognition so that the magnitude of unilateral or bilateral beamformer benefit might be predicted before the hearing aid fitting.

\section{Bilateral Beamformers}

Similar to unilateral beamformer benefits, investigators have documented bilateral beamformer benefits with high variability, which has been only partially explained by participant factors. For example, Best et al (2015) noted the range of bilateral beamformer benefit was approximately 50 percentage points and examined the potential for age and four-frequency puretone average (4FAPTA; average thresholds in $\mathrm{dB} \mathrm{HL}$ at $0.5,1.0,2.0$, and $4.0 \mathrm{~Hz}$ ) to be predictors of benefit. Using correlation analyses, the authors discovered a significant, positive relationship between 4FAPTA and bilateral beamformer benefit in one of the background noise conditions $(r=0.49$ and $r=0.48$ for the two beamformer implementations evaluated). These results suggest that listeners with larger degrees of hearing loss were more likely to benefit from the bilateral beamformer.

An individual listener's binaural hearing ability also might influence bilateral beamformer benefit. Bilateral beamformers disrupt binaural cues, even in commercial hearing aids (Picou et al, 2014). It is possible that participants who have good binaural hearing would be 
more negatively affected by the distortions and thus benefit less from a bilateral beamformer compared with participants who have poor spatial hearing abilities. One measure of binaural hearing is spatial release from masking (SRM), which is the calculated difference between (a) speech recognition performance measured with collocated speech and noise and (b) performance measured with spatially separated speech and noise. Although not exclusively dependent on spatial abilities (Best et al, 2012), the benefit from spatial separation of speech and noise is partly the result of an improvement in effective SNR due to interaural level differences (Zurek, 1993; Edmonds and Culling, 2006) and interaural timing differences (Zurek, 1993; Edmonds and Culling, 2005). Large SRM, as indicated by a large difference between performance in the collocated and separated conditions, can be interpreted as better binaural hearing because it indicates a listener is better able to take advantage of binaural cues to benefit from the spatial separation. However, the relationship between SRM and beamformer benefit has not been previously reported.

\section{Purpose}

The purpose of this study was to evaluate the effects of adaptive unilateral and adaptive bilateral beamforming in commercially available hearing aids on sentence recognition and subjective ratings in a laboratory environment. Participants were fitted with research hearing aids with three microphone settings (omnidirectional, adaptive unilateral beamformer, and adaptive bilateral beamformer) and were tested in two noise source configurations (side and surround) because noise source configuration has been shown to affect beamformer benefit (Ricketts and Henry, 2002; Hornsby and Ricketts, 2007). It was predicted that unilateral and bilateral beamformers would improve sentence recognition performance and subjective ratings, with additional advantages offered by the bilateral beamformer. A secondary purpose of this study was to evaluate potential participant factors related to beamformer benefits in the laboratory. Potential participant factors included age, degree of hearing loss, unaided speech recognition threshold in noise, unaided SRM, and omnidirectional performance in the experimental environment. It was expected that unaided speech in noise ability would predict unilateral beamformer benefit and that binaural hearing ability would predict additional advantage of the bilateral beamformer.

\section{MATERIALS AND METHODS}

\section{Participants}

Eighteen adults (50-80 yr, $M=66.2, \sigma=8.6$ ) completed the study. Six participants were female. All par- ticipants exhibited bilateral, symmetrical hearing loss, as defined by interaural asymmetries less than $20 \mathrm{~dB}$ at any audiometric frequency $250-4000 \mathrm{~Hz}$ and less than $15 \mathrm{~dB}$ at any three consecutive audiometric frequencies. Hearing losses were all sensorineural in nature, as evidenced by air-bone gaps $<15 \mathrm{~dB}$ at any audiometric frequency $500-4000 \mathrm{~Hz}$. Individual and mean audiometric thresholds, averaged across left and right ears, are displayed in Figure 1. Suspected hearing loss etiologies included hereditary $(\mathrm{N}=6)$, noise exposure $(\mathrm{N}=3)$, perinatal infection $(\mathrm{N}=1)$, unknown $(\mathrm{N}=1)$, combination of noise exposure and hereditary $(\mathrm{N}=4)$, or combination of noise and ototoxicity $(\mathrm{N}=3)$. No participant reported history of neurogenic disorder. Thirteen participants were experienced hearing aid users (2-25 yr, $M=6.0, \sigma=6.4$ ). No participants had previous experience with the hearing aid model used in the research study. The study was conducted with approval from Vanderbilt University's Institutional Review Board. Participants were compensated for their time at an hourly rate.

\section{Hearing Aid Fitting}

Participants were fitted with research hearing aids, Phonak Audéo receiver-in-the-canal instruments, with occluding, noncustom eartips. Hearing aids were programmed with three manually accessible programs, which varied only in their microphone setting. Microphone settings were (a) omnidirectional, (b) adaptive unilateral beamformer, and (c) adaptive bilateral beamformer. The omnidirectional microphone was approximately equally sensitive to sounds originating from all angles; the manufacturer-reported average directivity index was $-1.1 \mathrm{~dB}$. The adaptive unilateral beamformer is a traditional adaptive directional microphone setting, with a hypercardiod directivity pattern and a manufacturer-reported directivity index of $4.9 \mathrm{~dB}$. The adaptive bilateral beamformer had a manufacturer-reported directivity index of $6.9 \mathrm{~dB}$. The

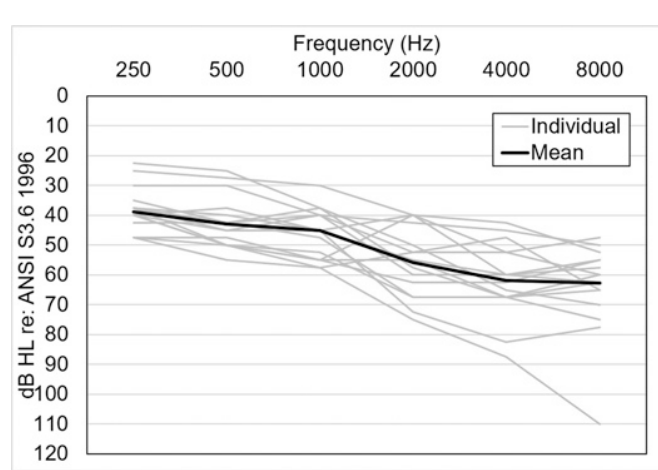

Figure 1. Mean audiometric thresholds averaged across ears for study participants (gray line) and the mean across participants (black line). 
bilateral beamformer preserved some binaural information by applying modeled interaural cues in a proprietary manner.

The hearing aids were programmed individually for each participant according to National Acoustics Laboratory's Nonlinear 2 prescriptive targets (Keidser et al, 2012). Match to target was verified for signals originating from $0^{\circ}$ using probe microphone measurements and a recorded speech passage using the Audioscan Verifit. Root mean square deviation from target across participants was less than $4 \mathrm{~dB}$ for all programs in both ears $(250-4000 \mathrm{~Hz})$. Advanced features were disabled during testing, including digital noise reduction, wind noise reduction, de-reverberation, and speech enhancement. Digital feedback reduction remained active.

\section{Conditions}

Participants were tested in a total of six conditions, which varied by microphone setting (omnidirectional, adaptive unilateral beamformer, adaptive bilateral beamformer) and by noise source configuration (side, surround). The "surround" configuration consisted of four loudspeakers, positioned $3.5 \mathrm{~m}$ surrounding the center of the participant position $\left(45^{\circ}, 135^{\circ}, 225^{\circ}\right.$, and $315^{\circ}$ ). The "side" configuration consisted of two loudspeakers, positioned $2.5 \mathrm{~m}$ from the center of the participant position at $90^{\circ}$ and $270^{\circ}$. Signal levels were adjusted in each condition such that the overall level was the same, despite the different number of loudspeakers. The level of the background noise in all conditions was either 63 or $68 \mathrm{dBA}$. The background noise level was chosen individually for each participant from these two levels to avoid performance in the ceiling or floor in the omnidirectional condition, as determined during a practice condition. Ten of the 18 participants were tested with a background noise level of $63 \mathrm{dBA}$. There was no significant difference in performance between participants tested in the 63 and 68 -dBA noise levels $\left[F_{(1,16)}=0.091, p=0.77, \eta_{\mathrm{p}}^{2}=0.01, M\right.$ difference $=1.9 \mathrm{RAU}, 95 \%$ confidence interval $(\mathrm{CI}):-15.2$ to 11.4$]$. The noise was an uncorrelated cafeteria babble with the same long term spectral average as the speech stimuli. Participants were tested in each condition twice. Participants were blinded to the research hypotheses and hearing aid test condition.

\section{Sentence Recognition}

Sentence recognition testing was conducted using the auditory-only passages from the Connected Speech Test (CST; Cox et al, 1987; 1988). The CST consists of 48 passages related to a specific topic (e.g., "window"). Each passage contains ten sentences with 25 key words for scoring. Before testing with a given passage, a participant was informed of the upcoming topic. Sentences were presented at 73-dB SPL from a loudspeaker directly in front of a participant. After each sentence, a participant verbally repeated as much of the sentence as possible. The experimenter manually scored the participant's verbal responses based on the key words in each sentence. Passages were always presented sequentially in pairs consistent with test instructions. Each condition was evaluated using one passage pair.

\section{Subjective Ratings}

Subjective ratings were acquired in each condition immediately following sentence recognition testing. Participants answered four questions on a scale of 0 to 10 , where 0 meant "very" and 10 meant "not at all." These questions, or a subset of these questions, have been used previously in laboratory-based investigations (Picou and Ricketts, 2014; Picou et al, 2017). The four questions were

- How hard did you work to understand what was said? Remember, this is different than how many words you got right. For example, you could get all the words right but have to work very hard to do it.

- How likely would you be to try to do something else to improve the situation (e.g., move to a quiet room, ask the speaker to speak louder)?

- How likely would you be to give up or just stop trying?

- How tired of listening do you feel?

The four questions, hereafter referred to as "work," "control," "give up," and "tired," were typed on a piece of paper with a horizontal number line below each question. The number lines included equally spaced demarcations from 0 to 10 with anchors at the extremes (very, not at all). Participants marked their responses on the same sheet of paper so their previous answers were visible, except they were provided a new ratings form after the completion of the first six conditions. Participants marked their responses in alphabetical order (e.g., an "A" for the first condition) to keep them blinded to the hearing aid condition and to avoid confusion with the numerical values used to anchor the questions.

\section{Unaided Speech in Noise}

Two clinical measures of speech understanding in noise were evaluated in all research participants, the Quick Speech in Noise Test (QuickSIN; Killion et al, 2004) and SRM using the Hearing in Noise Test (HINT; Nilsson et al, 1994). The QuickSIN is an adaptive, speech in noise test where sentences are presented at a fixed presentation level and the level of the background noise changes. Participants repeat the sentences and their responses are scored based on keywords in each sentence. For the 
purpose of this study, the QuickSIN was administered bilaterally via insert earphones at $70 \mathrm{~dB} \mathrm{HL}$, consistent with test instructions. Based on the number of keywords a participant successfully repeated, the SNR where they could understand $50 \%$ of the speech (SNR-50) was estimated according to the test instructions. QuickSIN thresholds were established for each participant using two lists from the group that have previously been shown to be equivalent (McArdle and Wilson, 2006). The scores from the two lists were averaged to reflect a single, bilateral QuickSIN score for each participant. The mean bilateral QuickSIN score for study participants was $8.2 \mathrm{~dB}$ (range $=0-20.5 \mathrm{~dB}, \sigma=6.4$ ).

The HINT was used to evaluate a participant's SRM. The HINT is an adaptive SNR test where the SNR required to achieve $50 \%$ speech understanding is estimated by varying the level of the speech in a fixed background noise level (65-dB SPL). Each HINT list contains ten sentences. The presentation level of the sentences is varied in 4-dB step sizes for four turnarounds and then 2-dB step sizes for the remainder of the list. The noise level is subtracted from the mean presentation level during the 2 -dB step size turnarounds to estimate an SNR. A single HINT score can be derived using ten sentences from one list or sentences from two lists presented consecutively. To evaluate the benefit of spatially separating the speech and noise, two lists of HINT sentences were presented consecutively in each of two speech and noise configurations, collocated, and separated. In the collocated condition, speech and noise originated from a loudspeaker located $1 \mathrm{~m}$ directly in front of a participant. In the separated condition, the speech signal originated $1 \mathrm{~m}$ directly in front of a participant and the noise originated from a loudspeaker positioned at $90^{\circ}$ (on the participant's left side). The mean HINT scores were $0.2 \mathrm{~dB}$ in the collocated condition ( range $=-5.1$ to $11.0, \sigma=3.5$ ) and $-0.8 \mathrm{~dB}$ in the separated condition (range $=-76$ to $8.8, \sigma=4.7)$. The difference between the SNR in the collocated and the separated conditions was used as an indication of a participant's SRM. The mean SRM score was $1.0 \mathrm{~dB}$ (range $=-4.4$ to $7.2, \sigma=3.1$ ).

\section{Procedure}

Following informed consent, participants underwent hearing testing using standard audiological procedures, in addition to the two unaided speech in noise measures (QuickSIN, HINT), in an audiometric sound booth. Then, the study hearing aids were fitted and verified in a quiet, clinic environment. After the fitting, data collection commenced in a moderately reverberant laboratory $(5.5 \times 6.5$ $\times 2.25 \mathrm{~m}$ ). Wall- and ceiling-mounted acoustic blankets were mounted on the front wall, back wall, and ceiling to achieve a moderate reverberation time (T30 $=708 \mathrm{msec}$ ). Speech stimuli were presented from a digital video disc player (Panasonic S54; Panasonic, Osaka, Japan), through a programmable attenuator (TDT System 3 PAG5) for level control, and finally to a loudspeaker (Tannoy System 600A; Tannoy, Coatbridge, Scotland) located $1.5 \mathrm{~m}$ directly in front of a participant. Noise signals were presented using Adobe Audition CS6 and routed through a sound card (Echo Layla 3G; Echo Digital Audio Corp, Santa Barbara, CA) to a multichannel amplifier (Crown CTs 8200; Harman, Elkhart, IN), and finally to unpowered loudspeakers (Tannoy System 600).

Figure 2 displays schematic diagram of the moderately reverberant test environment and loudspeaker configurations. Immediately before testing, a participant practiced with a CST passage not used for testing. This practice allowed the experimenter to determine if the background noise level needed to be lowered and to ensure the participant could perform the task. All participants started with $68-\mathrm{dB}$ background noise and it was changed to $63 \mathrm{~dB}$ if performance during practice was less than $20 \%$. Participants were tested a total of 12 times ( 3 hearing aid settings $\times 2$ noise configurations $\times 2$ repetitions). A participant completed testing with the three hearing aid microphone settings in one noise configuration before starting the second noise configuration. Then, testing was repeated in reverse order of noise configuration. Hearing aid condition test order was counterbalanced across participants, but stable within a participant. For example, a test order for one participant was: (a) omnidirectional with surround noise, (b) unilateral beamformer with surround noise, (c) bilateral beamformer with surround noise, (d) omnidirectional with side noise, (e) unilateral beamformer with side noise, (f) bilateral beamformer with side noise, (g) omnidirectional with side noise, (h) unilateral beamformer with side noise, (i) bilateral beamformer with

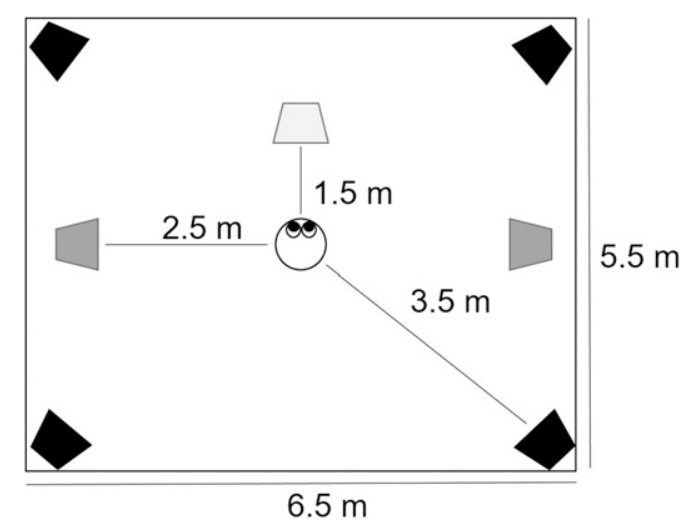

Figure 2. Loudspeaker arrangement in the moderately reverberant environment during testing. Black symbols indicate location of unpowered background noise loudspeakers during the "surround" noise source configuration conditions. Dark gray symbols indicate location of unpowered background noise loudspeakers during the "side" noise source configuration conditions. Light gray symbol indicates location of powered speech loudspeaker used during all testing. Distances are provided in meters. Figure is not to scale. 
side noise, (j) omnidirectional with surround noise, (k) unilateral beamformer with surround noise, and (l) bilateral beamformer with surround noise. CST passage pair was randomized across participants.

\section{RESULTS}

\section{Sentence Recognition}

Data from the two repetitions of each hearing aid setting and noise source configuration combination were averaged. Before analysis, the averaged data were converted to RAU to normalize the variance near the extremes (Studebaker, 1985). Figure 3 displays sentence recognition performance data for each hearing aid microphone setting; data are collapsed across noise configuration. Transformed sentence recognition scores were analyzed using generalized linear model with one random factor (participant) and two within-subject factors, hearing aid setting (omnidirectional, unilateral beamformer, bilateral beamformer), and noise source configuration (surround and side). Initial analyses also considered the noise level used for testing; however, results revealed the noise level did not affect the pattern of results. Thus, the noise level was not further considered in the analyses.

Analysis revealed a significant main effect of hearing aid microphone $\left[F_{(2,17)}=50.53, p<0.0001, \eta_{\mathrm{p}}{ }^{2}=0.86\right]$.
The main effect of noise configuration and the noise configuration $\mathrm{X}$ hearing aid microphone interaction were not significant $(p>0.10)$. Follow-up pairwise comparisons using planned linear contrasts revealed significant improvements in sentence recognition with unilateral beamformer relative to omnidirectional microphone $(p<0.001, M=27.76 \mathrm{RAU}, 95 \% \mathrm{CI}$ : 19.12-30.41), improvements with bilateral beamformer relative to omnidirectional ( $p<0.001, M=28.12 \mathrm{RAU}$, 95\% CI: 22.17-34.08), and additional benefits with bilateral relative to unilateral beamformer $(p<0.05$, $M=3.36 \mathrm{RAU}, 95 \% \mathrm{CI}: 0.29-6.43$ ). These data demonstrate a significant sentence recognition benefit with both unilateral and bilateral beamformers relative to the omnidirectional microphone and a small, but statistically significant, improvement in performance with the bilateral beamformer relative to the unilateral beamformer.

\section{Subjective Ratings}

Before analysis, the averaged data from each of the two repetitions in each condition were first reversed to facilitate data interpretation. After reversal, higher values indicate more work, stronger desire to control, more willingness to give up, and more tiredness. Reversed values were then converted to RAU to normalize

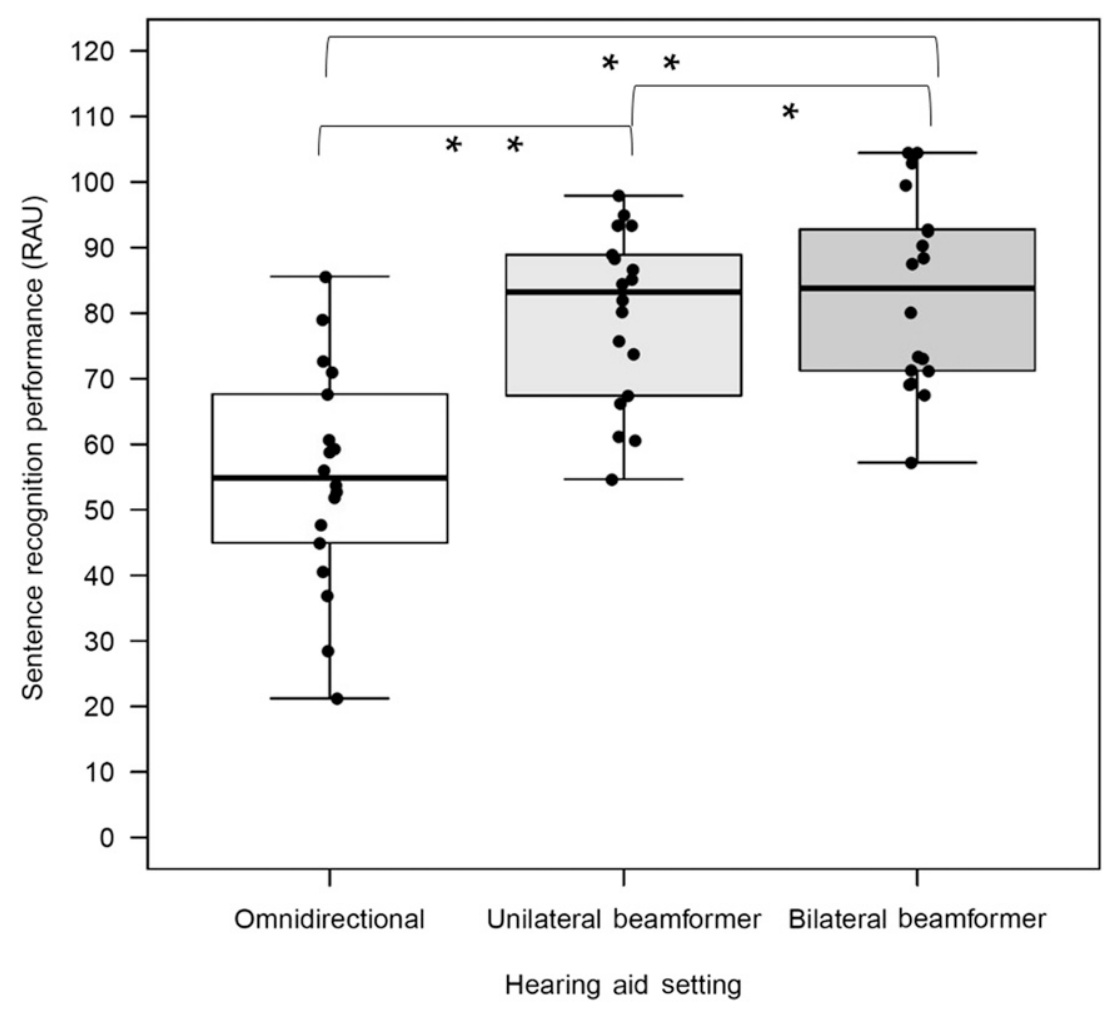

Figure 3. Sentence recognition performance (RAU) for each hearing aid microphone setting. The horizontal line within the box indicates the median. Boundaries of the box indicate the 25 th to 75 th percentile. The whiskers indicate the highest and the lowest values of the results. Individual data points are overlaid on the boxplots. $* *$ indicates significance at $p<0.01 ; *$ indicates significance at $p<0.05$. 
the variance near the extremes (Studebaker, 1985). Following transformation, data met assumptions of parametric analyses, as assessed by using Shapiro-Wilk test of normality on the studentized residuals $(p>$ $0.05)$ and Mauchly's test of sphericity $(p>0.05)$. As a result, ratings in response to each question were analyzed using generalized models, each with a single random factor (participant) and with two within-subject conditions, hearing aid setting (omnidirectional, unilateral beamformer, bilateral beamformer) and noise configuration (side, surround). Subjective ratings were inadvertently not recorded for one participant (71-yrold male) because of experimenter error. The following results are based on data from the remaining 17 participants. The results of all questions are displayed in Figure 4; data in this figure are collapsed across noise source configuration.

\section{Work}

Analysis of subjective ratings of work revealed a significant main effect of hearing aid condition $\left[F_{(2,16)}=\right.$ $\left.15.10, p<0.001, \eta_{\mathrm{p}}{ }^{2}=0.65\right]$. There was no main effect of noise configuration and no significant interaction. Planned comparisons revealed significant beamformer benefit relative to omnidirectional with both the unilateral beamformer ( $p<0.01, M=11.52 \mathrm{RAU}, 95 \% \mathrm{CI}$ : 5.70-17.34) and the bilateral beamformer $(p<0.001$, $M=14.03 \mathrm{RAU}, 95 \%$ CI: 8.61-19.44). The additional bilateral beamformer benefit was not statistically significant ( $p=0.21, M=2.51 \mathrm{RAU}, 95 \% \mathrm{CI}:-1.54$ to $6.55)$. These data demonstrate that participants reported working less hard to understand the speech with both types of beamformers, although ratings were comparable between the two beamformers.
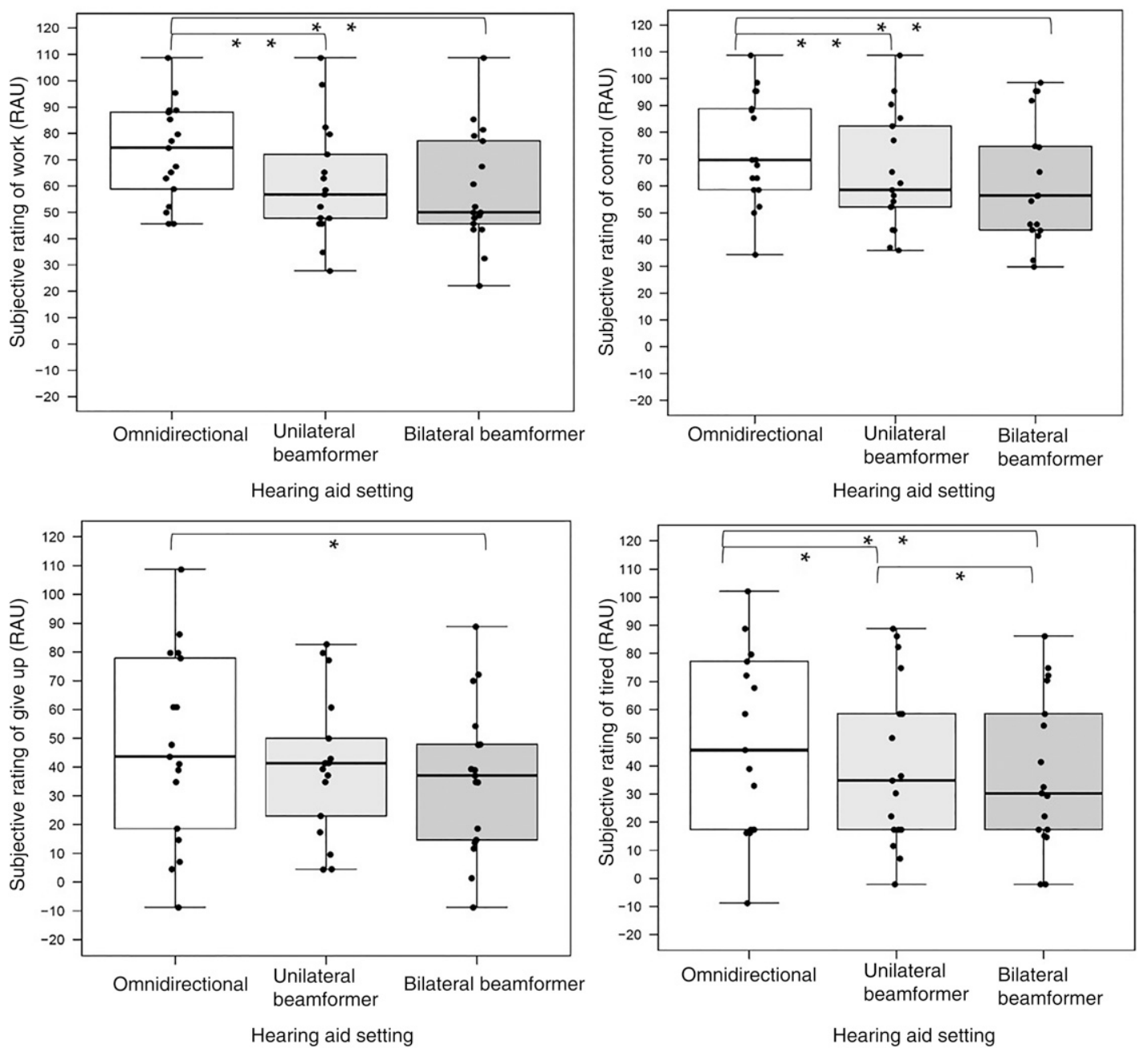

Figure 4. Mean subjective rating for questions of work (top left), control (top right), give up (bottom left), and tiredness (bottom right) with each of the microphone settings. Higher scores indicate higher reported need to work, higher desire to control the situation, higher likelihood of giving up, and more tiredness. The horizontal line within the box indicates the median. Boundaries of the box indicate the 25 th to 75 th percentile. The whiskers indicate the highest and the lowest values of the results. Individual data points are overlaid on the boxplots. ${ }^{*}$ indicates significance at $p<0.01 ; *$ indicates significance at $p<0.05$. 


\section{Control}

Analysis of subjective ratings of participants' desire to control the situation revealed a significant main effect of hearing aid condition $\left[F_{(2,16)}=7.84, p<0.01\right.$, $\left.\eta_{\mathrm{p}}{ }^{2}=0.50\right]$ and a significant effect of noise configuration $\left[F_{(2,16)}=4.78, p<0.05, \eta_{p}{ }^{2}=0.23\right]$. There was no significant interaction. Planned comparisons revealed significant beamformer benefit relative to omnidirectional with both the unilateral beamformer $(p<0.01, M=$ 8.71 RAU, 95\% CI: 3.93-14.03) and the bilateral beamformer ( $p<0.01, M=11.89$ RAU, 95\% CI: 5.53-18.25). The additional bilateral beamformer benefit was not statistically significant $(p=0.06, M=3.18 \mathrm{RAU}$, 95\% CI: -0.17 to 6.53$)$. These data demonstrate that participants reported less of a desire to control or improve the situation with the side configuration (5.09 RAU lower) and less of desire to control or improve the situation with either beamformer setting relative to the omnidirectional setting.

\section{Give Up}

Analysis of subjective ratings willingness to give up revealed a significant main effect of hearing aid condition $\left[F_{(2,16)}=4.92, p<0.05, \eta_{\mathrm{p}}{ }^{2}=0.40\right]$. There was no main effect of noise configuration and no significant interaction. Planned comparisons revealed a significant benefit only of the bilateral beamformer relative to omnidirectional ( $p<0.05, M=10.53 \mathrm{RAU}, 95 \% \mathrm{CI}: 2.82-$ 18.24). There was no unilateral benefit ( $p=0.26, M=$ 6.39 RAU, $95 \% \mathrm{CI}:-5.15$ to 17.93 ) or additional bilateral beamformer benefit ( $p=0.28, M=4.14$ RAU, $95 \%$ CI: -3.65 to 11.92 ). These data demonstrate that participants were less likely to give up or stop trying with the bilateral beamformer than in the omnidirectional condition.

\section{Tired}

Results of subjective ratings of tiredness revealed a significant main effect of hearing aid condition $\left[F_{(2,16)}=\right.$ 12.02, $\left.p<0.01, \eta_{\mathrm{p}}{ }^{2}=0.60\right]$. There was no main effect of noise configuration and no significant interaction. Planned comparisons revealed significant beamformer benefit relative to omnidirectional with both the unilateral beamformer ( $p<0.05, M=7.50 \mathrm{RAU}, 95 \% \mathrm{CI}: 0.38-$ 14.62) and the bilateral beamformer ( $p<0.01, M=$ 10.98 RAU, 95\% CI: 4.61-17.36). In addition, the additional bilateral benefit was significant ( $p<0.01, M=$ 3.48 RAU, 95\% CI: 0.91-6.05). These data demonstrate that participants reported feeling less tired with the bilateral beamformer than with the unilateral beamformer and feeling less tired with the unilateral beamformer than with the omnidirectional setting.

\section{Factors Related to Beamformer Benefit}

Hierarchical multiple regression was run to determine if participant factors could predict (a) unilateral beamformer benefit relative to performance in the omnidirectional condition and (a) additional bilateral beamformer benefit relative to performance in the unilateral beamformer condition. Participant factors were entered individually into the regression in the following order (a) participant age (in years), (b) 4FAPTA (0.5, 1.0, 2.0, and $4.0 \mathrm{kHz}$ ), (c) QuickSIN score (dB), (d) SRM score, and (e) performance in the omnidirectional condition (RAU).

Table 1 displays each regression model for unilateral beamformer benefit. The full model with all five participant factors (Model 5) was statistically significant $\left[R^{2}=\right.$ $0.85, F_{(5,35)}=15.64, p<0.001$, adj $\left.R^{2}=0.68\right]$. Table 2 displays regression coefficients for all participant factors in Model 5. Only two participant factors significantly increased $R^{2}$ : these were pure-tone average (PTA) $\left[R^{2}\right.$ change $\left.=0.25, F_{(1,33)}=11.19, p<0.01\right]$ and performance in the omnidirectional condition $\left[R^{2}\right.$ change $\left.=0.44, F_{(1,30)}=47.51, p<0.001\right]$. These results demonstrate that two participant factors, PTA and speech understanding in noise in the omnidirectional condition were significantly related to unilateral beamformer benefit (see Figure 5). For every $10 \mathrm{~dB}$ in additional hearing loss, participants benefited an additional 9 RAU from the unilateral beamformer. Conversely, for

Table 1. Results of Hierarchical Multiple Regression Investigating the Relationship between Participant Factors and Unilateral Beamformer Benefit

\begin{tabular}{|c|c|c|c|c|c|c|}
\hline Variables in Model & $R^{2}$ & Adjusted $R^{2}$ & Standard Error of the Estimate & $R^{2}$ Change & FChange & Significance of Change \\
\hline Model 1: age & 0.10 & -0.02 & 15.29 & 0.01 & 0.35 & 0.56 \\
\hline Model 2: age and PTA & 0.51 & 0.22 & 13.41 & 0.25 & 11.19 & $0.002^{\star \star}$ \\
\hline Model 3: age, PTA, and QuickSIN & 0.53 & 0.21 & 13.44 & 0.02 & 0.89 & 0.36 \\
\hline $\begin{array}{l}\text { Model 4: age, PTA, QuickSIN, and } \\
\text { SRM }\end{array}$ & 0.53 & 0.19 & 13.62 & 0.003 & 0.13 & 0.72 \\
\hline $\begin{array}{l}\text { Model 5: age, PTA, QuickSIN, } \\
\text { SRM, and performance in } \\
\text { omnidirectional }\end{array}$ & 0.85 & 0.68 & 8.61 & 0.44 & 47.51 & $<0.001^{\star *}$ \\
\hline
\end{tabular}

Note: PTA of $0.5,1.0,2.0,4.0 \mathrm{~Hz}$. 
Table 2. Regression Coefficients for All Variables in the Hierarchical Multiple Regression Model Regarding Unilateral Beamformer Benefit

\begin{tabular}{lcccc}
\hline Variable & $\begin{array}{c}\text { Unstandardized } \\
\text { Coefficient B }\end{array}$ & $\begin{array}{c}\text { Unstandardized Coefficient } \\
\text { Standard Error }\end{array}$ & $\begin{array}{c}\text { Standardized Coefficients Beta } \\
\text { Significance }\end{array}$ \\
\hline Age & -0.29 & 0.21 & -0.16 & 0.18 \\
PTA & 0.89 & 0.34 & 0.40 & $0.015^{\star}$ \\
QuickSIN & -1.03 & 0.47 & -0.43 & 0.04 \\
SRM & -0.58 & 0.49 & -0.13 & 0.24 \\
Performance in omnidirectional & -0.75 & 0.11 & -0.89 & $<0.001^{\star \star}$ \\
\hline
\end{tabular}

Note: PTA of $0.5,1.0,2.0,4.0 \mathrm{~Hz}$.

every $10 \mathrm{RAU}$ increase in speech in noise performance, participant unilateral beamformer benefit decreased by 7.5 RAU.

Table 3 displays each regression model and Table 4 displays the coefficients for the full model of additional bilateral beamformer benefit. None of the models were statistically significant. These results suggest that additional bilateral beamformer benefit was not related to any of the participant factors evaluated in the study. In total, these results demonstrate that people who performed well in the omnidirectional condition or had smaller degrees of hearing loss were less likely to benefit from unilateral beamformer. Conversely, participant factors did not account for significant variability in the models of additional bilateral beamformer benefit.

\section{DISCUSSION}

$\mathrm{T}$ he purpose of this study was to evaluate the effects of adaptive unilateral and adaptive bilateral beamforming in a commercially available hearing aid on sentence recognition and subjective ratings in a noisy laboratory environment. Based on the combination of findings that static bilateral beamformers can improve speech recognition and subjective ratings (Picou et al, 2014; Best et al, 2015) and that adaptive unilateral beamformers can provide larger benefits than static unilateral beamformers (Ricketts and Henry, 2002; Hornsby and Ricketts, 2007), it was expected that the bilateral beam- former would further improve sentence recognition and subjective ratings relative to the unilateral beamformer.

\section{Sentence Recognition}

Consistent with this hypothesis, results demonstrated statistically significant bilateral beamformer benefit for sentence recognition relative to both omnidirectional and unilateral beamformer microphones (see Figure 3). However, the additional bilateral beamformer benefit was small (3.4 RAU on average). Thus, the clinical benefit of this additional improvement warrants further investigation. Given the, at best, weak relationship between laboratory benefits and perceived field benefits of unilateral beamformers (Cord et al, 2004; Gnewikow et al, 2009; Wu, 2010), it is unclear if the average patient would notice the additional benefits in sentence recognition. However, the range of benefits in the present study was large ( -20 to $+29 \mathrm{RAU})$, so individual listeners might notice the additional benefits.

Somewhat surprisingly, the adaptive bilateral beamformer (3.4 RAU) did not reveal benefits as large as those previously reported with a static bilateral beamformer (12 RAU) under similar laboratory conditions (Picou et al, 2014). Methodological differences across studies limit the feasibility of making direct comparisons and drawing firm conclusions about the relative benefits of static versus adaptive bilateral beamformers [e.g., 73:63 speech to noise levels in the present study

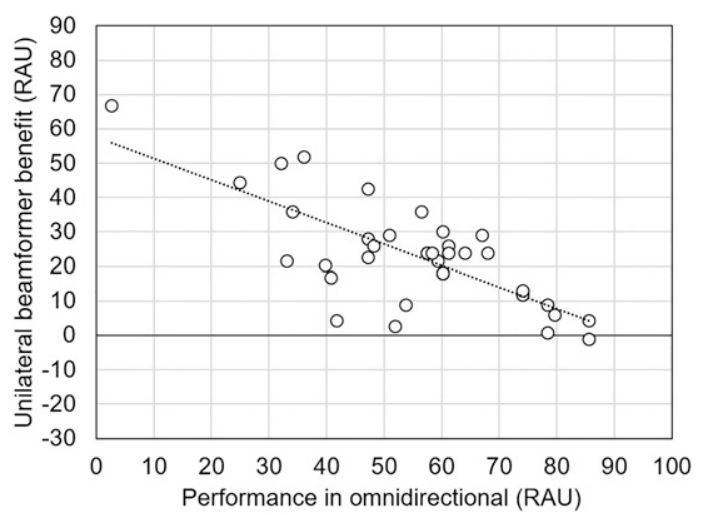

Figure 5. Calculated unilateral beamformer benefit as a function of PTA (left panel) and performance in omnidirectional condition (RAU; right panel). Both relationships are statistically significant $(p<0.05)$. 
Table 3. Results of Hierarchical Multiple Regression Investigating the Relationship between Participant Factors and Bilateral Beamformer Benefit

\begin{tabular}{|c|c|c|c|c|c|c|}
\hline Variables in Model & $R^{2}$ & Adjusted $R^{2}$ & $\begin{array}{l}\text { Standard Error } \\
\text { of the Estimate }\end{array}$ & $R^{2}$ Change & F Change & Significance of Change \\
\hline Model 1: age & 0.00 & -0.03 & 11.62 & 0.00 & 0.01 & 0.92 \\
\hline Model 2: age and PTA & 0.06 & 0.01 & 11.41 & 0.06 & 2.19 & 0.15 \\
\hline Model 3: age, PTA, and QuickSIN & 0.07 & -0.03 & 11.60 & 0.00 & 0.01 & 0.92 \\
\hline Model 4: age, PTA, QuickSIN, and SRM & 0.07 & -0.06 & 11.79 & 0.00 & 0.001 & 0.98 \\
\hline $\begin{array}{l}\text { Model 5: age, PTA, QuickSIN, SRM, and } \\
\text { performance in omnidirectional }\end{array}$ & 0.08 & -0.09 & 11.92 & 0.01 & 0.31 & 0.58 \\
\hline
\end{tabular}

Note: PTA of $0.5,1.0,2.0,4.0 \mathrm{~Hz}$.

compared with 72:69, 72:66, 65:56, and 65:53 levels in Picou et al (2014)]. Conversely, it is clear that the adaptive beamformer used in this study did not offer substantially improved benefits compared with those previously reported with static bilateral beamformers when the listener is surrounded by noise. Although speech recognition benefits for a static bilateral beamformer for noise sources only on the side have not been previously reported, no benefits would be expected relative to omnidirectional because these systems are typically not designed to have nulls to the sides. It is therefore important to note that the adaptive bilateral beamformer provided speech recognition benefits in these conditions that were similar to the adaptive unilateral beamformer.

It is also clear that unilateral beamformers can provide significant sentence recognition benefits in a laboratory (see Figures 3 and 5). This finding is consistent with a large body of evidence demonstrating unilateral beamformer benefits on sentence recognition at a fixed SNR with auditory-only stimuli (Walden et al, 2005; Wu and Bentler, 2010; Picou et al, 2014). This study expands these existing findings in part by also evaluating subjective ratings with both unilateral and bilateral beamformers.

\section{Subjective Ratings}

Consistent with the sentence recognition results, the subjective ratings of work, control, tiredness, and give up were better (lower) with the bilateral beamformer than with the omnidirectional microphone (Figure 4). The unilateral beamformer also improved ratings of work, control, and desire to give up. For the question of desire to give up (How likely would you be to give up or just stop trying?), only the bilateral beamformer provided significant benefits. Although the additional sentence recognition benefit of the bilateral beamformer was modest in this study, it seems that this additional improvement, perhaps combined with other undefined benefits, had a significant effect on participants' subjective experience of giving up. Of note, the bilateral beamformer significantly reduced desire to give up whereas the unilateral beamformer did not.

The bilateral beamformer also significantly decreased participants' tiredness (How tired of listening do you feel?) relative to both the omnidirectional and unilateral beamformer conditions. These data suggest that contrary to what one might expect given the small sentence recognition improvements, the bilateral beamformer processing improved the subjective participant experience in the laboratory in a measurable way. Recall that participants were blinded to the experimental conditions and research hypotheses. Thus, it is unlikely participants were influenced by the expected study outcomes. More work is warranted to comprehensively understand the phenomenon underlying participants' responses to ratings of tiredness and desire to give up.

For ratings of work and control, analysis revealed that both beamformers improved ratings relative to those with the omnidirectional microphone, but there was no additional benefit of the bilateral compared with the unilateral beamformer. These data suggest these two subjective ratings were insensitive to the modest additional benefits afforded by the bilateral beamformer.

Table 4. Regression Coefficients for All Variables in the Hierarchical Multiple Regression Model Regarding Additional Bilateral Beamformer Benefit

\begin{tabular}{lcccc}
\hline Variable & $\begin{array}{c}\text { Unstandardized } \\
\text { Coefficient B }\end{array}$ & $\begin{array}{c}\text { Unstandardized } \\
\text { Coefficient Standard Error }\end{array}$ & Standardized Coefficients Beta & Significance \\
\hline Age & -0.01 & 0.29 & -0.01 & 0.97 \\
PTA & -0.44 & 0.47 & -0.27 & 0.36 \\
QuickSIN & -0.13 & 0.66 & -0.07 & 0.85 \\
SRM & -0.05 & 0.68 & -0.01 & 0.95 \\
Performance in omnidirectional & -0.01 & 0.18 & -0.14 & 0.58 \\
\hline
\end{tabular}

Note: PTA of $0.5,1.0,2.0,4.0 \mathrm{~Hz}$. 


\section{Predictive Factors}

An additional purpose of this article was to evaluate participant factors that were related to beamformer benefit, either unilateral or bilateral. Potential factors investigated included age, degree of hearing loss, SNR loss, SRM, and performance in the omnidirectional condition. The range of unilateral and additional bilateral beamformer benefits in this study were both large ( -1 to $+67 \mathrm{RAU}$ and -20 to $+10 \mathrm{RAU}$, respectively). These benefit ranges are similar to previous investigations of unilateral beamformers (approximately 0-70 RAU; Wu and Bentler, 2010) and additional bilateral beamformer benefits ( -27 to +30 RAU; Best et al, 2015). Based on this range of benefits, it was expected that participant factors would be identified that would be related to calculated benefit.

There were no participant factors that predicted additional bilateral beamformer benefit. The best predictor of unilateral beamformer benefit was performance in the omnidirectional condition, accounting for $44 \%$ of the variance (see Tables 1 and 2). This finding is consistent with Ricketts and Mueller (2000), who combined data across three studies, all of which used adaptive sentence in noise task. Results indicated a weak relationship between omnidirectional performance and unilateral beamformer benefit; listeners with poorer performance benefited more from a unilateral beamformer. Because of the adaptive nature of the task, the results of that study were not limited by potential ceiling effects. It would have been mathematically possible for listeners with good performance to take advantage of unilateral beamformers. Combined, these data suggest that, in the laboratory, listeners who struggle more without beamforming microphones are the most likely to benefit from them, even without the confound of a ceiling effect.

In addition, PTA predicted unilateral beamformer benefit, accounting for $25 \%$ of the variability in the benefit scores (Table 1). People who have larger degrees of hearing loss were more likely to demonstrate unilateral beamformer benefit (see Figure 5, left panel). This finding is somewhat inconsistent with previous reports demonstrating that listeners with severe hearing loss demonstrate smaller unilateral beamformer benefits than listeners with moderate hearing loss (Ricketts et al, 2005; Ricketts and Hornsby, 2006). However, within this study sample of listeners with moderate hearing loss, those with more hearing loss and also more difficulty recognizing speech in the omnidirectional condition demonstrated larger benefits.

There was no relationship between unaided speech recognition (either SNR loss or SRM) or degree of hearing loss and benefit for either beamformer. Importantly, the results of this study do not support the notion that people with good spatial hearing or good speech in noise abilities are inherently poor candidates for bilateral beamforming technologies. The fact that SNR loss was not a significant predictor of benefit is somewhat surprising, given that omnidirectional performance was a significant predictor. These data suggest that individual frequency shaping is necessary to best predict speech recognition benefits for unilateral beamformers, precluding accurate clinical unaided predictions. Although the benefits of the bilateral beamformer evaluated in this study are quite small and variable, people who had good speech in noise abilities and good binaural hearing (as measured using SRM) were as likely to benefit as their peers who were more likely to struggle in noise and less likely to benefit from spatial separation of speech and noise. Like benefits from unilateral beamformers, more work is necessary to understand benefit factors that may affect clinical decisions to fit or not to fit bilateral beamformers.

\section{Study Limitations}

The results of this study suggest modest, but significant, additional benefits in sentence recognition and subjective ratings of tiredness and desire to give up with adaptive bilateral beamformers relative to a traditional unilateral beamformers. However, there are several study limitations that are worthy of mention. First, ceiling effects may have limited the noted benefits, particularly additional benefits of the bilateral beamformer. Inspection of Figure 5 reveals seven data points $>70$ RAU in the omnidirectional microphone setting. Although conversion to RAU from percent correct addresses this limitation statistically, it becomes mathematically difficult to demonstrate large benefits with performance near the ceiling. The nature of the fixed SNR test used in this study lends itself to the potential from problematic ceiling effects. However, fixed SNR tests may be preferable than adaptive ones for within-participant investigations of hearing aid technologies (Naylor, 2016). Regardless, future work is warranted to further explore the magnitude of expected and realized benefits, particularly of the bilateral beamformer in adverse listening conditions.

A second study limitation is participant heterogeneity. Although all participants were $>50 \mathrm{yr}$ and met inclusion criteria for bilateral, symmetrical, mild to moderately severe hearing loss, they reflected a range of hearing loss etiologies. The heterogeneity is evident, in part, in Figure 5, which demonstrates a broad range of sentence recognition scores in the omnidirectional condition. The variability in speech recognition performance is consistent with previous work demonstrating significant variability in performance even within conditions of similar audibility (Sherbecoe and Studebaker, 2003). Exploratory analysis revealed that performance in the omnidirectional condition could not be explained by noise level, age, or hearing aid experience. 
Only 13 participants were experienced hearing aid users and none had experience with the technology under investigation. Although previous investigations suggest hearing aid experience does not influence unilateral or bilateral beamformer benefits for laboratory speech in noise paradigms (Picou et al, 2017), the possibility that hearing aid experience influenced benefits in this study was explored. Although, the small sample of non-hearing aid users in the study precluded full analysis, exploratory analysis in the current data set revealed a trend for experienced users to benefit more from the unilateral beamformer $(\sim 17$ RAU more benefit) but less than the bilateral beamformer $(\sim 4$ RAU). Thus, the interaction between hearing aid experience and beamformer benefit warrants further study.

Finally, the study sample size may have been too small to fully realize the relationships between the participant factors and beamformer benefits. Based on the regression analyses presented in Tables 1 and 3, estimated sample sizes would need to be approximately 100 to have sufficient power to detect some of the participant factors as significant. Future work will be necessary to explore these participant factors and beamformer benefit with a larger sample to detect the more subtle relationships that might exist between participant factors and benefit.

\section{CONCLUSIONS}

$\mathrm{T}$ he results of the study confirm and extend previous findings of bilateral beamformer benefits to include conditions with side originating noise with an adaptive beamformer. Noted benefits were generally modest (3.4 RAU), suggesting the need to determine realistic expectations for additional improvements patients might expect with bilateral beamformer microphones. However, the results of this investigation did find notable improvements in subjective ratings of tiredness and desire to give up with the bilateral beamformer, even relative to the unilateral beamformer. The combination of these results suggests that the measured benefits in sentence recognition performance with the bilateral beamformer were large enough to be noticed by the participants and were large enough to affect how they were feeling during testing. These subjective benefits may be related to cognitive benefits of the technology, sound quality, or some other factor not explored in the study, all of which warrant further investigation. Finally, although none of the variability in additional bilateral beamformer benefits could be accounted for by participant factors, unilateral benefit was associated with worse performance in the omnidirectional condition and larger degrees of hearing loss, suggesting those who needed the most help after optimizing audibility through amplification were the most likely to derive benefit.
Acknowledgments. The authors thank Maddox Myers, Sunaina Sherchan, and Anna Allen for their assistance in participant recruitment and data collection, as well as Travis Moore for his assistance with the figures.

\section{REFERENCES}

Aspell E, Picou E, Ricketts T. (2014) Directional benefit is present with audiovisual stimuli: limiting ceiling effects. $J$ Am Acad Audiol 25(7):666-675.

Bentler R, Palmer C, Mueller HG. (2006) Evaluation of a secondorder directional microphone hearing aid: I. Speech perception outcomes. J Am Acad Audiol 17(3):179-189.

Bentler RA, Egge JL, Tubbs JL, Dittberner AB, Flamme GA. (2004) Quantification of directional benefit across different polar response patterns. J Am Acad Audiol 15(9):649-659, quiz 660.

Best V, Marrone N, Mason CR, Kidd G, Jr. (2012) The influence of non-spatial factors on measures of spatial release from masking. $J$ Acoust Soc Am 131(4):3103-3110.

Best V, Mejia J, Freeston K, van Hoesel RJ, Dillon H. (2015) An evaluation of the performance of two binaural beamformers in complex and dynamic multitalker environments. Int $J$ Audiol 54(10):727-735

Blamey PJ, Fiket HJ, Steele BR. (2006) Improving speech intelligibility in background noise with an adaptive directional microphone. J Am Acad Audiol 17(7):519-530.

Compton-Conley CL, Neuman AC, Killion MC, Levitt H. (2004) Performance of directional microphones for hearing aids: realworld versus simulation. J Am Acad Audiol 15(6):440-455.

Cord MT, Surr RK, Walden BE, Dyrlund O. (2004) Relationship between laboratory measures of directional advantage and everyday success with directional microphone hearing aids. J Am Acad Audiol 15(5):353-364.

Cornelis B, Moonen M, Wouters J. (2012) Speech intelligibility improvements with hearing aids using bilateral and binaural adaptive multichannel Wiener filtering based noise reduction. $J$ Acoust Soc Am 131(6):4743-4755.

Cox RM, Alexander GC, Gilmore C. (1987) Development of the connected speech test (CST). Ear Hear 8(5 Suppl):119S-126S.

Cox RM, Alexander GC, Gilmore C, Pusakulich KM. (1988) Use of the connected speech test (CST) with hearing-impaired listeners. Ear Hear 9(4):198-207.

Dhar S, Humes LE, Calandruccio L, Barlow NN, Hipskind N. (2004) Predictability of speech-in-noise performance from real ear measures of directional hearing aids. Ear Hear 25(2):147-158.

Edmonds BA, Culling JF. (2005) The spatial unmasking of speech: evidence for within-channel processing of interaural time delay. $J$ Acoust Soc Am 117(5):3069-3078.

Edmonds BA, Culling JF. (2006) The spatial unmasking of speech: evidence for better-ear listening. J Acoust Soc Am 120(3):1539-1545.

Gnewikow D, Ricketts T, Bratt GW, Mutchler LC. (2009) Realworld benefit from directional microphone hearing aids. J Rehabil Res Dev 46(5):603-618.

Hornsby BW, Ricketts TA. (2007) Effects of noise source configuration on directional benefit using symmetric and asymmetric directional hearing aid fittings. Ear Hear 28(2):177-186. 
Humes LE, Wilson DL. (2003) An examination of changes in hearing-aid performance and benefit in the elderly over a 3-year period of hearing-aid use. J Speech Lang Hear Res 46(1):137-145.

Jespersen CT, Olsen S. (2003) Does directional benefit vary systematically with omnidirectional performance? Hear Rev 10:16-25.

Keidser G, Dillon H, Carter L, O’Brien A. (2012) NAL-NL2 empirical adjustments. Trends Amplif 16(4):211-223.

Keidser G, Dillon H, Convery E, Mejia J. (2013) Factors influencing individual variation in perceptual directional microphone benefit. J Am Acad Audiol 24(10):955-968.

Killion MC, Christensen LA. (1998) The case of the missing dots: $\mathrm{Al}$ and SNR loss. Hear J 51(5):32-34.

Killion MC, Niquette PA, Gudmundsen GI, Revit LJ, Banerjee S. (2004) Development of a quick speech-in-noise test for measuring signal-to-noise ratio loss in normal-hearing and hearing-impaired listeners. J Acoust Soc Am 116(4 Pt 1):2395-2405.

Kochkin S. (2000) MarkeTrak V: "why my hearing aids are in the drawer": the consumers' perspective. Hear J 53(2):34, 36, 39-41.

Kochkin S. (2010) MarkeTrak VIII: consumer satisfaction with hearing aids is slowly increasing. Hear $J$ 63:19-20.

Kompis M, Dillier N. (1994) Noise reduction for hearing aids: combining directional microphones with an adaptive beamformer. $J$ Acoust Soc Am 96(3):1910-1913.

Kompis M, Dillier N. (2001) Performance of an adaptive beamforming noise reduction scheme for hearing aid applications. II. Experimental verification of the predictions. J Acoust Soc Am 109(3):1134-1143.

Kuk F, Keenan D, Lau C-C, Ludvigsen C. (2005) Performance of a fully adaptive directional microphone to signals presented from various azimuths. J Am Acad Audiol 16(6):333-347.

Lotter T, Vary P. (2006) Dual-channel speech enhancement by superdirective beamforming. EURASIP J Appl Signal Process 2006:1-14.

McArdle RA, Wilson RH. (2006) Homogeneity of the 18 QuickSIN[TM] lists. J Am Acad Audio 17(3):157-167.

McCormack A, Fortnum H. (2013) Why do people fitted with hearing aids not wear them? Int $J$ Audiol 52(5):360-368.

Naylor G. (2016) Theoretical issues of validity in the measurement of aided speech reception threshold in noise for comparing nonlinear hearing aid systems. J Am Acad Audiol 27(7):504-514.

Nilsson M, Soli SD, Sullivan JA. (1994) Development of the hearing in noise test for the measurement of speech reception thresholds in quiet and in noise. J Acoust Soc Am 95(2):1085-1099.

Picou EM, Aspell E, Ricketts TA. (2014) Potential benefits and limitations of three types of directional processing in hearing aids. Ear Hear 35(3):339-352.

Picou EM, Moore TM, Ricketts TA. (2017) The effects of directional processing on objective and subjective listening effort. $J$ Speech Lang Hear Res 60(1):199-211.

Picou EM, Ricketts TA. (2014) Increasing motivation changes subjective reports of listening effort and choice of coping strategy. Int $J$ Audiol 53(6):418-426.

Picou EM, Ricketts TA. (2017) How directional microphones affect speech recognition, listening effort and localisation for listeners with moderate-to-severe hearing loss. Int J Audiol 56(12):909-918.
Picou EM, Ricketts TA, Hornsby BW. (2013) How hearing aids, background noise, and visual cues influence objective listening effort. Ear Hear 34(5):e52-e64.

Plomp R. (1986) A signal-to-noise ratio model for the speech-reception threshold of the hearing impaired. J Speech Hear Res 29(2): $146-154$.

Preves DA, Sammeth CA, Wynne MK. (1999) Field trial evaluations of a switched directional/omnidirectional in-the-ear hearing instrument. J Am Acad Audiol 10(5):273-284.

Ricketts T, Dhar S. (1999) Comparison of performance across three directional hearing aids. J Am Acad Audiol 10(4):180-189.

Ricketts T, Henry P. (2002) Evaluation of an adaptive, directionalmicrophone hearing aid. Int $J$ Audiol 41(2):100-112.

Ricketts T, Mueller HG. (2000) Predicting directional hearing aid benefit for individual listeners. J Am Acad Audiol 11(10):561-569, quiz 575

Ricketts TA, Henry PP, Hornsby BW. (2005) Application of frequency importance functions to directivity for prediction of benefit in uniform fields. Ear Hear 26(5):473-486.

Ricketts TA, Hornsby BW. (2003) Distance and reverberation effects on directional benefit. Ear Hear 24(6):472-484.

Ricketts TA, Hornsby BWY. (2006) Directional hearing aid benefit in listeners with severe hearing loss. Int J Audiol 45(3):190-197.

Sherbecoe RL, Studebaker GA. (2002) Audibility-index functions for the connected speech test. Ear Hear 23(5):385-398.

Sherbecoe RL, Studebaker GA. (2003) Audibility-index predictions of normal-hearing and hearing-impaired listeners' performance on the connected speech test. Ear Hear 24(1):71-88.

Studebaker GA. (1985) A "rationalized" arcsine transform. $J$ Speech Hear Res 28(3):455-462.

Taylor B. (2003) Speech-in-noise tests: how and why to include them in your basic test battery. Hear $J$ 56(1):40-43.

Valente M, Fabry DA, Potts LG. (1995) Recognition of speech in noise with hearing aids using dual microphones. J Am Acad Audiol 6(6):440-449.

Walden BE, Surr RK, Cord MT. (2003) Real-world performance of directional microphone hearing aids. Hear $J$ 56(11):40-42.

Walden BE, Surr RK, Grant KW, Van Summers W, Cord MT, Dyrlund O. (2005) Effect of signal-to-noise ratio on directional microphone benefit and preference. $J$ Am Acad Audiol 16(9): $662-676$.

Welker DP, Greenberg JE, Desloge JG, Zurek PM. (1997) Microphone-array hearing aids with binaural output. II. A twomicrophone adaptive system. IEEE Trans Speech Audio Process 5: 543-551.

Wu Y-H. (2010) Effect of age on directional microphone hearing aid benefit and preference. J Am Acad Audiol 21(2):78-89.

Wu YH, Bentler RA. (2010) Impact of visual cues on directional benefit and preference: part I-laboratory tests. Ear Hear 31(1): $22-34$.

Zurek PM. (1993) Binaural advantages and directional effects in speech intelligibility. In: Studebaker GA, Hochberg I, eds. Acoustical Factors Affecting Hearing-Aid Performance. Boston, MA: Allyn \& Bacon, 255-276. 